\title{
Nutrición Enteral Domiciliaria (NED) en niños y adolescentes. Recomendaciones de la Rama de Nutrición de la Sociedad Chilena de Pediatría
}

\author{
Home Enteral Nutrition (NED) in children and adolescents. \\ Recommendations of the Nutrition Branch of the Chilean Society of Pediatrics \\ María Luisa Cordero B. ${ }^{a}$, María Isabel Hodgson B. ${ }^{b}$, Karin Walewska Schilling F.c, \\ Salesa Barja Y. ${ }^{\text {b,d }}$, Eliana Muñoz B. ${ }^{e}$, Rosita Antilef H. ${ }^{f}$
}

\author{
aServicio de Pediatría Hospital Dr. Sótero del Río \\ bDepartamento de Gastroenterología y Nutrición, División de Pediatría, Facultad de Medicina, Pontificia Universidad Católica de Chile. \\ 'Servicio de Pediatría Hospital Dr. Roberto del Río \\ dServicio Pediatría Hospital Josefina Martínez de Ferrari \\ eServicio Pediatría Hospital Luis Calvo Mackenna \\ 'Servicio Pediatría Hospital San Borja Arriarán \\ En representación de la Rama de Nutrición de la Sociedad Chilena de Pediatría, SOCHIPE
}

Recibido: 3 de diciembre de 2018; Aceptado: 7 de diciembre de 2018

\section{Resumen}

El uso de apoyo nutricional ambulatorio, enteral o parenteral, ha sido un paso necesario en la optimización del soporte nutricional en pacientes, que, por diversas patologías, no logran cumplir con sus requerimientos por vía oral (VO). En el presente artículo se presentan las recomendaciones de la Rama de Nutrición, dirigidas a los equipos de salud que atienden pacientes pediátricos, que requieran alimentación enteral por un tiempo prolongado. Su objetivo general es entregar pautas para un correcto manejo en estos pacientes. Se describe la conformación ideal del equipo de salud para atención y seguimiento de dichos pacientes, los criterios de ingreso al programa y su forma de evaluación en el tiempo. Además, se describen características generales de la alimentación enteral, vías de administración, fórmulas enterales disponibles, complicaciones de este soporte nutricional y por último monitorización y seguimiento del paciente.

\section{Abstract}

The use of home enteral or parenteral nutrition has been a necessary step in the optimization of nutritional support in patients who, due to several diseases, fail to meet their nutritional requirements by oral feeding. This article presents the recommendations of the Chilean Pediatric Society Nutritional Branch, aimed at health teams that treat pediatric patients who require enteral feeding for a long
Palabras clave: Alimentación enteral domiciliaria; soporte nutricional; fórmulas enterales; sondas enterales; ostomías

\section{Keywords:}

Home enteral nutrition; nutritional support; enteral formulas; enteral tube feeding; ostomy 
time. The general objective is to provide guidelines for the proper management of these patients. It describes the ideal conformation of the health team for the care and follow-up of those patients, the program admission criteria, and its evaluation method over time. In addition, it describes general characteristics of enteral feeding, routes of administration, available enteral formulas, complications, and patient follow-up.

\section{Introducción y justificación}

El uso de apoyo nutricional ambulatorio, enteral o parenteral, ha sido un paso necesario en la optimización del soporte nutricional en pacientes, que, por diversas patologías, no logran cumplir con sus requerimientos por vía oral $(\mathrm{VO})^{1}$. En la última década, los programas de asistencia nutricional domiciliaria (AND), han demostrado ser seguros y efectivos en el manejo de pacientes de alta complejidad ${ }^{2}$. Los avances tecnológicos y el desarrollo de equipos multidisciplinarios con experiencia en manejo nutricional han permitido que pacientes hospitalizados, que requieren soporte nutricional por períodos prolongados, puedan ser dados de alta para continuar recibiendo este apoyo en su hogar. Esta modalidad de tratamiento mejora la calidad de vida del paciente y de su familia, disminuye las complicaciones propias de hospitalizaciones prolongadas, libera camas de hospital y reduce costos. Estudios a nivel mundial señalan que el costo de tratamiento disminuiría entre un $65-80 \%{ }^{2}$. Además, el reintegrar al paciente a su hogar le permite retomar ciertas actividades propias de su edad, como la escolarización ${ }^{3}$. En Chile, la nutrición enteral domiciliaria (NED) se realiza en la mayoría de los centros pediátricos y actualmente, desde enero 2017, está financiada por la ley Ricarte Soto, Ley $\mathrm{N}^{\circ} 20.850$.

Las indicaciones más frecuentes de NED en pediatría corresponden a niños(as) con trastornos de deglución de diferente origen y a niños(as) con patología crónica que no logran cubrir requerimientos por VO, presentando incremento ponderal insuficiente.

Esta recomendación de la Rama de Nutrición va dirigida a los equipos de salud que atienden pacientes pediátricos beneficiarios del sistema de salud chileno, que requieran alimentación enteral por un tiempo prolongado, mayor a 3 meses. Su objetivo general es entregar pautas para un correcto manejo en estos pacientes. Los objetivos específicos son:

1. Identificar los pacientes pediátricos candidatos a NED.

2. Describir los requisitos necesarios para administrar Nutrición Enteral (NE) en el domicilio.

3. Definir los profesionales que deben integrar el equipo multidisciplinario, especificando sus roles.
4. Precisar los parámetros necesarios para la monitorización clínica, nutricional y metabólica de estos pacientes.

5. Prevenir e identificar precozmente las complicaciones de la terapia nutricional.

6. Reintegrar al paciente a su hogar y entorno social.

Para implementar la NED se requiere un equipo multidisciplinario encargado de evaluar las necesidades del paciente, certificar si cumple con los requisitos establecidos para ingresar al programa, indicar el soporte nutricional requerido y efectuar el seguimiento con evaluaciones periódicas ${ }^{4,5}$.

El equipo debiera estar conformado por:

1. Pediatra o Médico de familia: responsable del cuidado integral del niño. Debe mantenerse en estrecho contacto con el nutriólogo tratante, revisar en conjunto el plan de acción y coordinar las intervenciones y evaluaciones por otros especialistas involucrados en la atención del paciente.

2. Pediatra Nutriólogo: responsable de evaluar la condición clínica del paciente previo a la derivación al domicilio, establecer el plan de tratamiento y definir las indicaciones de Nutricion Parenteral (NP) y/o NE según el caso. Además, debe realizar controles seriados y reevaluación periódica para ajustar las indicaciones nutricionales.

3. Enfermero/a: debe evaluar integralmente al paciente y a su familia y realizar educación según protocolo. Certificar si cumple con criterios de ingreso al programa, establecer necesidades de insumos, revisar condiciones del hogar en conjunto con asistente social (AS) y supervisar la puesta en marcha y el seguimiento del programa.

4. Nutricionista $(\mathrm{N})$ : debe participar en la evaluación nutricional y prescripción del plan de alimentación, colaborar en la educación del paciente y su familia, y efectuar seguimiento y registro en forma periódica, incluyendo visitas en domicilio.

5. Asistente social (AS): responsable de evaluar las condiciones socioeconómicas del grupo familiar, las características de la vivienda, la existencia de redes de apoyo y planificar estrategias de acción específica. 
6. Psicólogo/a: responsable de evaluar la condición psicológica del paciente y de sus cuidadores previo al alta hospitalaria, anticipar el impacto del tratamiento domiciliario a nivel familiar, identificar condiciones emocionales que puedan interferir en la evolución del paciente y establecer necesidades de terapia o derivación.

En algunos casos, según la patología de base del paciente, complicaciones o intercurrencias, será necesario contar con el apoyo de otros profesionales.

Para ingresar al programa de NED el paciente y su cuidador deben cumplir con ciertos requisitos:

1. Paciente clinicamente estable, con tolerancia y eficacia comprobada a la alimentación prescrita.

2. Domicilio en condiciones adecuadas, certificado por visita domiciliaria.

3. Haber completado programa de educación realizado por la enfermera a uno o dos cuidadores y al paciente cuando corresponda.

4. Haber realizado reunión de equipo multidisciplinario con énfasis en la revisión de todos los requerimientos (check-list).

5. Haber firmado el Consentimiento informado, tanto el apoderado, como el profesional responsable de educación, y el asentimiento, cuando corresponda.

La educación y entrenamiento de uno o dos cuidadores debe contemplar dos aspectos fundamentales: entrega de contenidos teóricos y adquisición de habilidades. La entrega de contenidos y el lenguaje de la enseñanza, debe adecuarse al nivel educativo, nivel de comprensión y edad de los cuidadores. El desarrollo de habilidades debe realizarse mediante la práctica simulada de los procedimientos, tantas veces como se requiera, hasta lograr la administración al paciente de la NE en forma segura ${ }^{6}$. La enfermera responsable de la capacitación entrenará al menos a un cuidador en los conocimientos teóricos y prácticos para la administración segura de la fórmula enteral por sonda u ostomía y en el uso de bomba de infusión, cuando se requiera. Una vez entrenado el cuidador, se procederá a aplicar la técnica en el paciente bajo la supervisión de enfermera, usando una pauta de cotejo.

Previo al inicio de NED, se requiere una visita domiciliaria por AS o enfermera, para verificar las condiciones físicas del hogar e identificar las adecuaciones necesarias. En casos puntuales deberá realizarse una segunda visita, para certificar en terreno si las recomendaciones se implementaron

La evaluación del programa de NED debe incluir:

1. Supervisión periódica del cumplimiento de las pautas de alimentación.

2. Valoración de los resultados nutricionales del programa.

3. Notificación y registro de complicaciones y eventos adversos.

\section{Nutrición Enteral Domiciliaria (NED)}

La administración de nutrientes por vía digestiva en la casa del paciente permite manejar una gran variedad de patologías y situaciones nutricionales (tabla 1). La NE se puede entregar a nivel gástrico o intestinal. Para decidir la vía de acceso más adecuada en cada caso, es importante considerar el diagnóstico, la anatomía y funcionalidad del tracto gastrointestinal, el tiempo programado de apoyo nutricional, el riesgo de aspiración y el nivel de actividad física (figura 1) ${ }^{7,8}$. La decisión de la vía de acceso es una indicación médica. Ésta puede ser mediante sondas u ostomías. La sonda vía nasal es la vía utilizada con mayor frecuencia. Está indicada cuando el tiempo necesario de apoyo nutricional es menor de 2-3 meses. Puede asociarse a complicaciones locales, pero el uso de sondas flexibles y el

Tabla 1. Principales indicaciones de Nutrición Enteral Domiciliaria

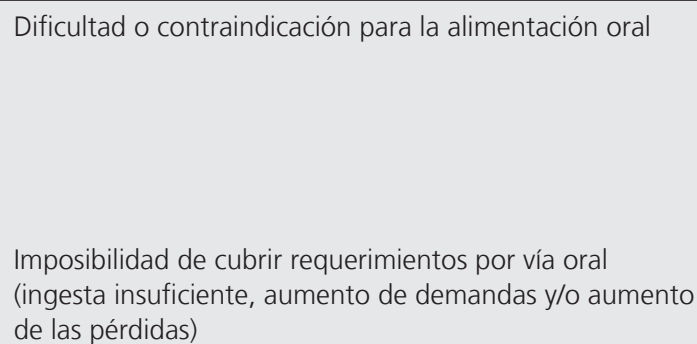

de las pérdidas)

\author{
Enfermedades neurológicas \\ Trastornos de deglución \\ Inmadurez de los mecanismos de deglución (prematurez) \\ Patología esofágica \\ Malformaciones oro-faciales \\ - Cardiopatías \\ - Enfermedad Renal Crónica \\ - Hepatopatías crónicas \\ Fibrosis Quística \\ Enfermedad Inflamatoria Intestinal
}

Transición de NP a vía oral 


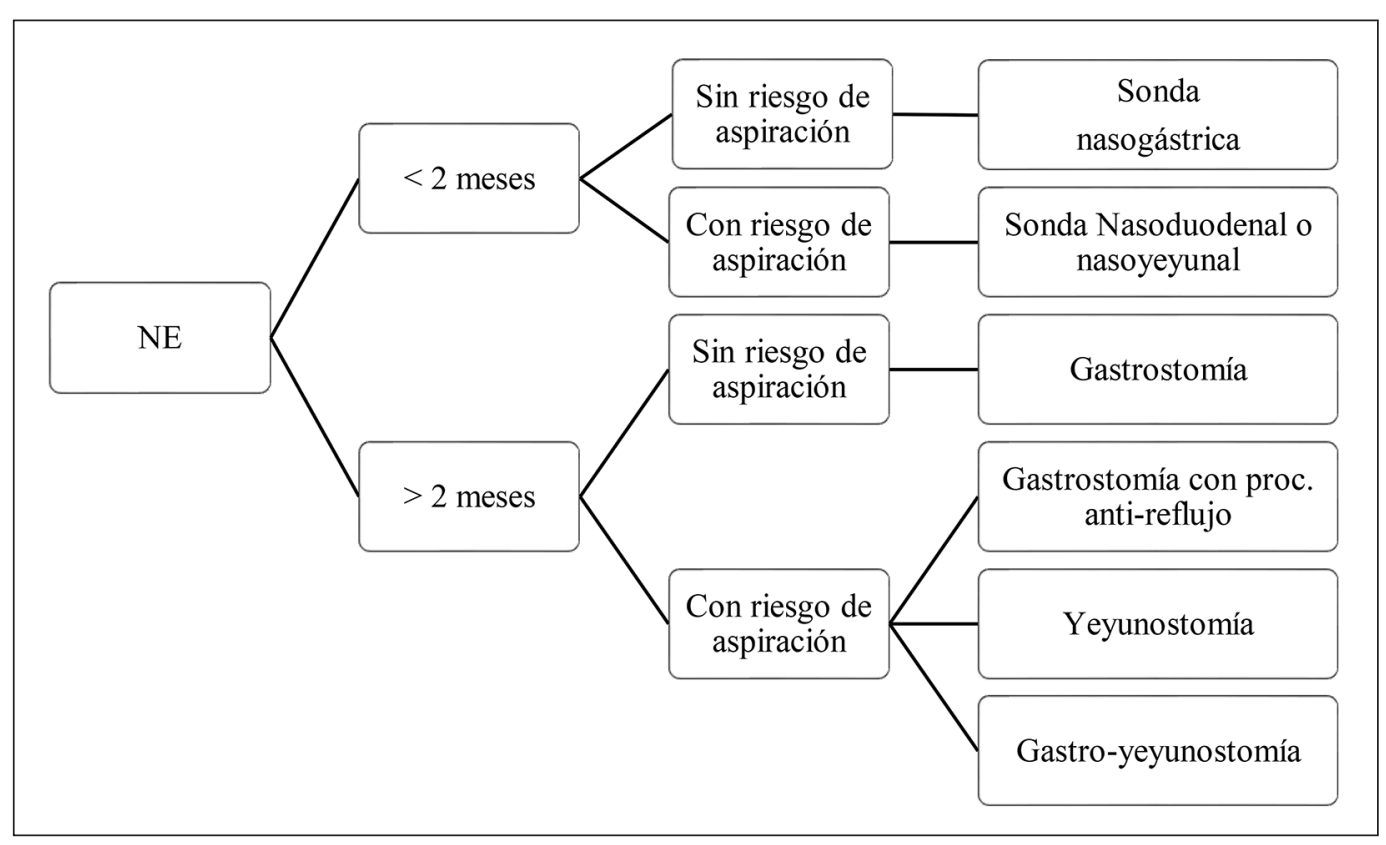

Figura 1. Algoritmo para la selección de la vía de acceso para la Nutrición Enteral (Adaptado de: Boullata J y col.) adecuado cuidado de enfermería mejoran la tolerancia y disminuyen las complicaciones. Según la ubicación del extremo distal puede ser nasogástrica o nasoenteral (transpilórica). La nasogástrica es de fácil instalación y monitorización; adecuada para la infusión continua, intermitente por bolo o ciclada, diurna y/o nocturna. Puede producir distensión gástrica y tiene riesgo de aspiración a la vía aérea. La nasoenteral o transpilórica (duodenal o yeyunal) está indicada en pacientes con riesgo de aspiración (reflujo gastroesofágico, trastorno de deglución, gastroparesia, alteración de conciencia). Evita la distensión gástrica, pero es más difícil de instalar y mantener en posición. Puede facilitar el reflujo duodeno-gástrico, requiere control radiológico y obliga al uso de infusión continua con bomba de alimentación.

La ostomía es la vía de elección en pacientes que requieren NE prolongada (más de 2-3 meses). Puede ser gástrica o yeyunal. La gastrostomía es el acceso más frecuente, tiene ventajas frente a las sondas naso-enterales, permite mayor movilidad del paciente, afecta menos la imagen corporal y al tener mayor calibre permite administrar alimentación mixta. Está contraindicada cuando hay gastroparesia, hipertensión portal o cirugías previas que dificultan la movilización gástrica. Su instalación puede ser quirúrgica o endoscópica percutánea (GEP). Ésta última requiere de una hospitalización más breve, tiene menor costo y menos complicaciones, pero no permite realizar simultáneamente el procedimiento antirreflujo. La yeyunostomía quirúrgica es de uso infrecuente en pediatría, está indicada cuando existe riesgo de aspiración o trastornos que comprometan la motilidad gástrica. Implica mayor dificultad para su instalación y seguimiento. Requiere uso de bomba de alimentación para infusión continua. La gastro-yeyunostomía se logra al avanzar la sonda distalmente, desde la gastrostomía hacia la zona postpilórica, pudiendo quedar un segundo lumen en estómago, lo cual permite la descompresión de este. La técnica de instalación es dificultosa.

Los cuidados de enfermería son importantes para minimizar las molestias provocadas por las sondas $\mathrm{u}$ ostomías y prevenir complicaciones asociadas a su uso. Están destinados a mantener la sonda en buenas condiciones, evitar su desplazamiento, mantener un adecuado aseo de las cavidades naso-bucales, evitar lesiones de piel y mucosas, y administrar medicamentos con las precauciones necesarias para evitar obstrucción de la sonda.

La elección de la fórmula enteral (FE) corresponde al equipo de nutrición. Se realiza después de una completa evaluación que considera edad, estado nutricional, antecedentes de intolerancia o alergia alimentaria, vía de administración, enfermedad de base, e indemnidad anatómica y funcional del tubo digestivo. Estos antecedentes permiten estimar los requerimientos hídricos y los de macro y micronutrientes para definir la FE más adecuada ${ }^{9}$. Actualmente existen preparados en polvo para ser reconstituidos, lo que permite realizar cambios en su concentración o adicionar suplementos, y fórmulas líquidas RTH, del inglés ready to hang. Éstas últimas son de mayor costo, pero más seguras en su composición, tienen menor riesgo de contaminación y de exceder la osmolalidad tolerable a nivel intestinal. 
La osmolalidad de las fórmulas pediátricas oscila entre 220 y $450 \mathrm{mOsm} / \mathrm{kg}$. Las FE diseñadas para adultos, en general no se recomiendan en menores de 10 años, porque su aporte proteico excede los requerimientos de éstos, el de micronutrientes suele ser insuficiente, y aportan una mayor carga renal de solutos. Existen también en el mercado FE pediátricas con densidad energética de $1,5 \mathrm{Kcal} / \mathrm{ml}$, pero por su alta osmolalidad se deben utilizar con precaución. En aquellos pacientes con restricción de volumen, permiten alcanzar buen aporte calórico. Algunos factores que pueden afectar la tolerancia a las FE son: osmolalidad elevada, distensión abdominal, vómitos, diarrea, enfermedades intercurrentes, sobrecrecimiento bacteriano y medicamentos entregados a través de la misma vía ${ }^{8}$.

Una alternativa más económica a las FE completas es la alimentación mixta, utilizando fórmulas lácteas convencionales complementadas con preparados caseros de sopas mixtas licuadas y enriquecidas. Éstas contienen mayor cantidad de fibra, favoreciendo el tránsito y el trofismo intestinal. Deben administrarse por ostomía de calibre apropiado para evitar dilución inadecuada. Su uso por SNG o SNE está contraindicado.

\section{Complicaciones de la NED}

Éstas pueden ser de la vía de alimentación utilizada, gastrointestinales y/o metabólicas. Las complicaciones de la vía de alimentación pueden ser a su vez de las sondas naso-enterales o de las ostomías. Las primeras se refieren a mala posición secundaria a desplazamiento o a una obstrucción. Se previenen con una buena fijación y con lavados de la sonda post alimentación, respectivamente. La obstrucción de la sonda puede manejarse con bebidas carbonatadas o preparados enzimáticos. Las lesiones por decúbito constituyen otra complicación de las sondas (erosión del cartílago nasal, esofagitis, gastritis); pueden minimizarse utilizando sondas siliconadas, cambiando frecuentemente el punto de apoyo nasal y lubricando con suero fisiológico. Las complicaciones más frecuentes de las ostomías son infecciones cutáneas y granuloma peri-ostomía, peritonitis química por extravasación de alimento o atascamiento del borde interno de la gastrostomía en la mucosa gástrica (Buried bumper síndrome). Éstas últimas deben comunicarse al equipo tratante o ser evaluadas en servicio de urgencia.

Las complicaciones gastrointestinales como regurgitación, vómitos, diarrea o distensión abdominal son de causa multifactorial (mal posición de la sonda, velocidad de infusión acelerada, uso de fórmulas con alta osmolalidad o contaminadas). El manejo es variable según la etiología que las produzca. Se debe evaluar el uso de antibióticos cuando se sospecha disbacteriosis o contaminación de la fórmula. Cuando el problema es constipación, se sugiere aumentar aporte de agua y fibra dietaria. Las complicaciones metabólicas pueden producirse por un desequilibrio entre aportes y requerimientos o debido a alguna intercurrencia. Para evitarlas o pesquisarlas precozmente, es importante el control periódico clínico y de laboratorio, para realizar los ajustes pertinentes de macro y micronutrientes. Durante el transcurso de la NED pueden ocurrir complicaciones graves como íleo paralítico, obstrucción intestinal, peritonitis e isquemia intestinal que obligan a suspender la NE hasta que se resuelva la intercurrencia.

La transición desde la NE a la vía oral es un proceso que debe ser individualizado y no siempre ocurre ${ }^{11}$. En este proceso influye principalmente la duración de la alimentación enteral, los mecanismos de succióndeglución y la patología de base, siendo más fácil si se han realizado las medidas de prevención. Para efectuar el traslape se requiere cumplir los siguientes criterios:

1. Patología de base resuelta o estable.

2. Estado nutricional compensado.

3. Desarrollo de succión-deglución suficiente y segura, avalado por informe fonoaudiológico y/o estudio de imágenes cuando se requiera.

4. Presencia de un cuidador capacitado.

Las técnicas para favorecer la transición se resumen en la figura $2^{12,13}$. En el período de transición a la VO se pueden presentar trastornos alimentarios de etiología mixta: orgánicos, postraumáticos y/o conductuales. Se deben sospechar frente a síntomas como náuseas, vómitos, cólicos o inapetencia, al intentar la alimentación oral. Para prevenirlos, es útil realizar estimulación oral y succión no nutritiva en forma precoz. Algunos casos requieren apoyo profesional con fonoaudiólogo y/o terapeuta ocupacional ${ }^{9,10}$.

\section{Monitorización y seguimiento de los pacientes con NED}

La monitorización y seguimiento de los pacientes debe considerar la tolerancia, la respuesta del paciente, la evolución de los parámetros previamente alterados y la aparición de complicaciones metabólicas asociadas a su uso. El mejor marcador de adecuación de la NE es el buen estado nutricional. Es necesario evaluar tolerancia enteral, descartando presencia de distensión abdominal, náuseas o vómitos, y observando frecuencia y características de las deposiciones, chequear regularmente el estado de la vía de alimentación, la posición de la sonda y detectar complicaciones locales. Evaluar crecimiento mediante parámetros antropométricos (peso, talla, perímetro craneano) y sus índices respectivos y registrar su evolución gráfica. La medición adicional de 


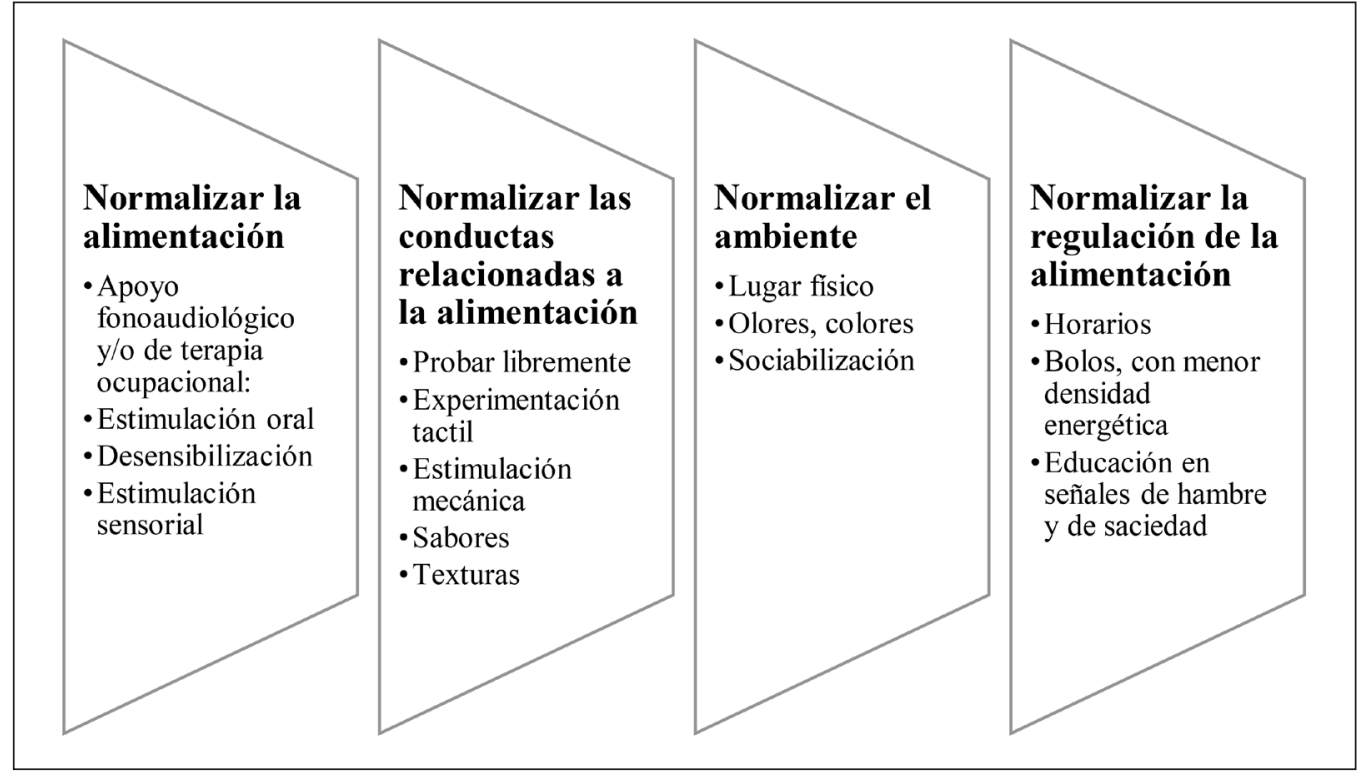

Figura 2. Técnicas para normalizar la alimentación (adaptado de Schauster $\mathrm{H}$ ). pliegues cutáneos y perímetro braquial, permite valorar masa grasa y masa magra. Además, debe efectuarse control periódico de parámetros de laboratorio (gases venosos, electrolitos, hemograma, perfil bioquímico, función renal y hepática, ferritina, $25 \mathrm{OH}$ vitamina D) y otras mediciones más específicas según requiera el paciente ${ }^{14}$.

\section{Conclusiones}

En Chile, la nutrición enteral domiciliaria se indica y realiza actualmente en la mayoría de los centros pediátricos y desde enero 2017, cuenta con financiamiento a través de la Ley $\mathrm{N}^{\circ} 20.850$, conocida como Ley Ricarte Soto. La Rama de Nutrición de la Sociedad Chilena de Pediatría, ha tenido un rol activo en la implementación de este proyecto ley y actualmente, en la revisión y supervisión de los pacientes que postulan e ingresan a dicho programa. En este artículo se preten- de poner énfasis en los puntos que consideramos de mayor relevancia para una correcta indicación, manejo y supervisión de los pacientes pediátricos con NED.

\section{Conflicto de intereses}

Los autores declaran no tener conflicto de interés.

\section{Agradecimientos}

Agradecemos a todos los miembros de la Rama de Nutrición de la Sociedad Chilena de Pediatría y a los equipos de nutrición de los Hospitales Luis Calvo Mackenna, Roberto del Río, San Borja Arriaran, Félix Bulnes, San Juan de Dios, Sótero del Río, Padre Hurtado, Josefina Martínez y Universidad Católica, quienes participaron en forma activa en la elaboración de estas recomendaciones.

\section{Referencias}

1. Dudrick SJ., O’Donnell JJ., Englert DM. et al. 100 patient-years of ambulatory home total parenteral nutrition. Ann Surg. 1984;199(6):770-81.

2. Marshall JK., Gadowsky SL., Childs A., Armstrong D. Economic analysis of home vs hospital-based parenteral nutrition in Ontario, Canada. J Parenter Enteral Nutr. 2005;29(4):266-9.
3. Norman JL., Crill CM. Optimizing the transition to home parenteral nutrition in pediatric patients. Nutr Clin Pract. 2011;26(3):273-85.

4. Koletzko B, Goulet O, Hunt J, Krohn K, Shamir R; Parenteral Nutrition Guidelines Working Group. Guidelines on paediatric parenteral nutrition of the European Society of Paediatric Gastroenterology, Hepatology and Nutrition (ESPGHAN) and the European Society for Clinical
Nutrition and Metabolism (ESPEN), supported by the European Society of Paediatric Research (ESPR). J Pediatr Gastroenterol Nutr. 2005;41 Suppl 2:S187.

5. Dewan T., Cohen E. Children with medical complexity in Canada. Paediatr Child Health. 2013;18(10):51822.

6. Gifford H, Delegge M. Epperson LA. Education methods and techniques 
for training home parenteral nutrition patients. Nutr Clin Pract. 2010;25(5):44350.

7. Barja S. Capítulo 37. Nutrición Enteral. En: Gana JC, Harris P, Hodgson MI. Ed. Práctica clínica en Gastroenterología, Hepatología y Nutrición Pediátrica. Primera edición. Editorial Ediciones UC. Santiago de Chile, 2015. Pág. 389-400.

8. Boullata J., Nieman Carney L., Guenter P. Enteral Formulas IN ASPEN Enteral Nutrition Handbook. Silverspring, 2010. MD: ASPEN.

9. Corrales K, Bechard L, Kane K, Kelleher
D. Enteral Nutrition. Chapter 16. In: Hendricks K, Duggan C, Walker W. Manual of Pediatric Nutrition, $3^{\circ}$ Ed. 2000. BC Decker. Hamilton. London.

10. Pinelli J, Symington A. Non-nutritive sucking for promoting physiologic stability and nutrition in preterm infants. 2001; The Cochrane Library.

11. Angulo D, Bustos E, Sánchez A, Barja S. Rehabilitación de la alimentación por vía oral en niños con enfermedades respiratorias crónicas y nutrición enteral prolongada. Nutr Hosp. 2016;33(4):368-.
12. Schauster H, Dwyer J. Transition from tube feedings to feedings by mouth in children: Preventing eating dysfunction. J Am Diet Assoc. 1996;96(3):277-81.

13. Kindermann A, Kneepkens CM, Stok A, van Dijk EM, Engels M, Douwes AC. Discontinuation of tube feeding in young children by hunger provocation. J Pediatr Gastroenterol Nutr. 2008;47(1):87-91.

14. Braegger C, Decsi T, Dias JA, et al. Practical approach to paediatric enteral nutrition: a comment by the ESPGHAN Committee on Nutrition. J Pediatr Gastroenterol Nutr. 2010;51(1):110-22. 